\title{
Methicillin-resistant Staphylococcus aureus (MRSA) among dental patients: a problem for infection control in dentistry?
}

\author{
Melanie Zimmerli • Andreas F. Widmer • Marc Dangel • \\ Andreas Filippi • Reno Frei • Jürg Meyer
}

Received: 15 September 2008 / Accepted: 8 December 2008 /Published online: 23 December 2008

(C) Springer-Verlag 2008

\begin{abstract}
We assessed the frequency of carriers of methicillinresistant Staphylococcus aureus (MRSA) among 500 dental patients of a university clinic. From each participant, two specimens were taken from the anterior nares and the pharynx and analysed by culture. The participants completed a questionnaire on possible risk factors of MRSA infection. Two hundred ten individuals carried S. aureus, 90 in the nares only, 51 in the throat only and 69 in nares and throat. Isolates of 208 patients were methicillin-sensitive; two isolates were methicillin-resistant, both carried in the throat exclusively. In conclusion, the frequency of nasal and/or throat carriers of MRSA among dental patients was low and suggests few opportunities of exposure in the dental clinic assessed.
\end{abstract}

Keywords Staphylococcus aureus · Methicillin resistance · MRSA carriers · Epidemiology $\cdot$ Infection control

\section{Zimmerli · A. Filippi}

Department of Oral Surgery, Oral Radiology and Oral Medicine, School of Dental Medicine, University of Basel,

Basel, Switzerland

A. F. Widmer · M. Dangel

Hospital Epidemiology, University Hospital Basel,

Basel, Switzerland

R. Frei

Laboratory of Diagnostic Bacteriology, University Hospital Basel, Basel, Switzerland

\section{J. Meyer $(\bowtie)$}

Institute of Preventive Dentistry and Oral Microbiology,

School of Dental Medicine, University of Basel,

Hebelstrasse 3,

4056 Basel, Switzerland

e-mail: juerg.meyer@unibas.ch

\section{Introduction}

Staphylococcus aureus is an important bacterial pathogen causing a wide variety of infections ranging from mild local infections of skin and soft tissue to severe systemic infections such as sepsis and toxic shock syndrome, which may be lethal. Typically, this bacterium lives as a commensal in the nose and/or throat of $20 \%$ to $70 \%$ of adults [17, 31]. Methicillin-resistant S. aureus (MRSA) which are generally resistant to multiple antibiotics have emerged among persons in hospitals, nursing homes and other health care institutions since the 1960s and are called hospital- or health care-associated MRSA (HA-MRSA) [2]. They account for a large part of nosocomial infections worldwide [29, 33] and are associated with longer hospitalisation and higher lethality [37]. Most of these nosocomial infections are caused by few successful epidemic clones of HA-MRSA [20].

In the community setting, several outbreaks of severe infections with MRSA have been reported among people lacking established risk factors for HA-MRSA acquisition [6]. The bacteria are referred to as community-associated MRSA (CA-MRSA) and differ from HA-MRSA in several important aspects $[9,44]$. First reported in the 1980s, these CA-MRSA infections seem to have increased in prevalence in the past several years, affecting children and adult populations as diverse as aboriginal communities in Australia, military recruits, individuals involved in contact team sports, prisoners or intravenous drug users [22, 44].

CA-MRSA caused predominantly skin and soft tissue infections [32], but was also associated with severe pulmonary infections including fatal necrotising pneumonia and empyema [24]. Recent increases in CA-MRSA infections in the USA appear to be associated with a few strains of $S$. aureus [22], some of which have recently disseminat- 
ed in Denmark [27], whilst other European isolates were found to be genetically more diverse [3, 15].

In hospitals, MRSA are transmitted by indirect or direct contact mainly, but transmission by droplets or rarely airborne dispersal has been observed among health care workers [36]. Many hospitals have successfully introduced concerted measures to reduce HA-MRSA prevalence, including meticulous hand disinfection after every patient contact as well as early detection of carriers and their decontamination [5, 11, 43]. Guidelines and/or surveillance strategies have been set up at national levels [19, 41]. Direct contact is also the most common mode of transmission of CA-MRSA.

Prevention efforts in specific populations and circumstances should optimally be guided by epidemiological data not available in the dental setting. MRSA proportion in hospitals varies considerably among countries: about $1 \%$ in the Nordic countries and The Netherlands, $>40 \%$ in southern Europe (Portugal, Italy, Turkey) [14, 42] and $25 \%$ to $>60 \%$ in the USA [33]. Switzerland exhibits a low prevalence of MRSA in hospitals (on the average 2\%), but the distribution is heterogeneous (Geneva $>30 \%$ MRSA) $[4,16]$.

The aim of this study was to determine the MRSA carrier rate among dental patients as representation of the Basel general population by a prospective prevalence study allowing targeted infection control in dental practice.

\section{Materials and methods}

From August to November 2006, 500 volunteers were recruited among the adult patients of the Department of Oral Surgery, Dental School, University of Basel. The only exclusion criterion was age $<18$ years. The test persons were informed on the study verbally and in writing and gave written informed consent. The study had been approved by the Basel State Ethical Committee (protocol no. 232/04). All participating individuals completed a questionnaire including personal data and medical history as well as questions on possible risk factors for MRSA carriage.

Two specimens were obtained with sterile polyester fibre-tipped swabs moistened with sterile saline from the anterior nares (three rotations in each anterior nostril) and from the posterior wall of the pharynx. The samples were taken by a dentist who had been appropriately trained.

Swabs were brought to the microbiology laboratory in a transport medium (Venturi Transystem, Copan, Italy). Within $4 \mathrm{~h}$, the swabs were inoculated into a selective enrichment broth (brain heart infusion broth with $6 \% \mathrm{NaCl}$, Axonlab, Baden, Switzerland). After incubation at $35^{\circ} \mathrm{C}$ overnight, the broth was subcultured on both a chromogenic agar for $S$. aureus (S. aureus ID Agar, bioMérieux, France) and a blood agar plate (Columbia with $5 \%$ sheep blood, Becton Dickinson, Heidelberg, Germany) for $24 \mathrm{~h}$.

Colonies suspicious of $S$. aureus were identified on the basis of several traits such as typical colonies on the chromogenic agar and blood agar plus presence of clumping factor, protein A and capsular antigens as determined by an agglutination test (Slidex Staph Plus, bioMérieux, France). Methicillin sensitivity of all $S$. aureus isolates was examined by agar diffusion on Mueller-Hinton agar using a cefoxitin disk according to the guidelines of the Clinical and Laboratory Standards Institute [21]. Data analyses were performed using SPSS software (SPSS, Chicago, USA). Level of significance was $p<0.05$.

\section{Results}

More than $90 \%$ of the patients asked participated voluntarily in the study. The median age was 48.5 years (range 18 91 years) among the 500 individuals screened. The gender of participants was equally distributed (women $49.2 \%$, men $50.8 \%$ ).

S. aureus was isolated from 210 test persons $(42 \%$; Table 1). Of particular interest is the group of 51 individuals $(10.2 \%)$ who were exclusive throat carriers. Isolates of 208 individuals $(41.6 \%)$ were sensitive to methicillin (MSSA), whilst two throat isolates $(0.4 \%)$ were MRSA (Table 1). Data from the two MRSA carriers, a woman and a man, are shown in Table 2. The number of MRSA carriers was too small to find statistically significant associations. Both MRSA-positive participants were offered a successful decolonisation treatment [8], but neither of them responded to attempts by multiple telephone calls and letters.

\section{Discussion}

The prevalence of colonisation with $S$. aureus in this population of dental patients (42\%) was in the range

Table 1 S. aureus carrier rates among the 500 dental patients studied

\begin{tabular}{lc}
\hline & $n(\%)$ \\
\hline S. aureus carriage, overall & $210(42 \%)$ \\
Nasal carriage & $159(31.9 \%)$ \\
Throat carriage & $120(24 \%)$ \\
Nasal and throat carriage & $69(13.9 \%)$ \\
Exclusive nasal carriage & $90(18 \%)$ \\
Exclusive throat carriage & $51(10.2 \%)$ \\
MSSA carriage & $208(41.6 \%)$ \\
MRSA carriage in throat & $2(0.4 \%)$ \\
\hline
\end{tabular}


Table 2 Characteristics of the two MRSA carriers

\begin{tabular}{lll}
\hline Sex & Male & Female \\
\hline Age (years) & 25 & 29 \\
Nationality & Swiss & Swiss \\
Other people living in the same household & 2 & 2 \\
Reason for visiting the Department of Oral Surgery & Pain because of apical & Pain because of apical \\
& periodontitis & periodontitis \\
Suffering from chronic disease & No & Epilepsy \\
History of skin soft tissue infection & No & No \\
Hospitalisation in the past 12 months & No & 4 days (gynaecology) \\
Antibiotic treatment in the past 12 months & No & No \\
Ever used drugs (iv or inhaled) & No & No \\
Playing a contact sport & No & No \\
Accommodation in a camp in the past year & No & No \\
\hline
\end{tabular}

determined in other populations in Europe and in the USA [33]. It was slightly lower than in other collectives examined in the Basel region with closer ties to the health care system (i.e. blood donors $54 \%$, health care workers 48\%) [31].

The anterior nares are known to be the primary colonisation site of $S$. aureus [18]. In this study, 51 people, i.e. $10.2 \%$ of those tested and $24.2 \%$ of the carriers, harboured S. aureus exclusively in the throat. In addition, the two MRSA-positive individuals carried these staphylococci in the throat, not in the nares. These findings support the postulate that screening for $S$. aureus carriers should include swabs from nares and throat to improve detection [31]. Recently, it has been suggested that colonisation sites other than the anterior nares play an important role in the development of CA-MRSA infection; genital without nasal colonisation has also been reported [10].

The MRSA prevalence among individuals seeking dental treatment in Basel is low $(0.4 \%)$ as has been reported $(0.2-$ $1.3 \%$ ) among community members [35]. However, MRSA is the most common pathogen in US emergency rooms in patients with skin and soft tissue infections. Therefore, infection control activities should be initiated before the problem of CA-MRSA becomes endemic. In fact, Geneva already experienced the first outbreak of CA-MRSA in the community [28].

The number of MRSA carriers in this population was too low to draw firm conclusions with respect to risk factors for CA-MRSA colonisation. However, both MRSA carriers were young, appeared to be immunologically competent and had few contacts to the health care system in the past 12 months, which was thought to be typical [9, 32], but may apply to only a subset of CA-MRSA infections [12]. However, these criteria have been defined based on infections, and to our knowledge, it has not been established if they are applicable to carriers as well. We had intended to genotype the two isolates by determining the spa and pulsed-field types in order to compare them to the genotypes prevalent in the nearby university hospital [15]. Unfortunately, both isolates were lost by accident and re-isolation was not possible because the two carriers did not respond to the offers of decolonisation treatment.

Carriage of MRSA in the nose and/or throat of patients or dentists may not represent the only reservoir for transmission within the dental office. Recent data suggested that presence of $S$. aureus in the oral cavity may be more frequent than previously thought [13, 39]. The proportion of MRSA among 1,017 S. aureus isolates from 5,005 oral specimens was $6 \%$ in a study from Scotland [40], and $S$. aureus (sensitivity to methicillin not determined) has recently been linked to peri-implant infections in a study from Sweden [34].

MRSA are most frequently spread via transiently contaminated hands of health care professionals, but contaminated surfaces and objects may play a minor role in MRSA transmission [26]. Infection control in the dental practice is regulated by national guidelines [1, 7, 23, 25]. These hygiene measures are designed to safeguard the health and safety of both patients and staff, in particular to prevent the transmission of blood-borne infections, primarily hepatitis B or C viruses, as well as infectious agents spread by contact or droplets, e.g. Mycobacterium tuberculosis. Standard precautions are considered effective to prevent MRSA transmission to the dental team and from patient to patient [7, 26, 38]. In particular, strict adherence to hand disinfection before and after every patient contact and wearing personal protection equipment (gloves, mask, gown, eye protection) are recommended [1, 38].

Nevertheless, as long as the MRSA carrier rates in the general population are low and infection control measures are followed strictly, we consider visit to a dentist not as a risk factor for the spreading of CA-MRSA. Other risk factors appear dominant [35]. However, dentists themselves may become a source of transmission. To our knowledge, there is only documented transmission of MRSA from a dentist whose nares had been colonised to two patients in 
England [30]. However, this had occurred before standard infection control practices were instituted in that practice [30].

In conclusion, the frequency of nasal and/or throat carriers of MRSA among dental patients in Basel, Switzerland was low. The results suggest few opportunities of exposure in the dental office. However, more data are needed to evaluate the epidemiology of MRSA and their role in the healthy and diseased oral cavity.

Conflict of interest The authors declare that they have no conflict of interest.

\section{References}

1. Baehni P, Dotesio D, Ferrari E, Guggenheim B, Meyer J, Mombelli A, Roh S (2005) Praxishygiene, Qualitätsleitlinien in der Zahnmedizin, 2nd edn. Schweiz Monatschr Zahnmed 115 (no. 7), pp 15-24

2. Barrett FF, McGehee RF Jr, Finland M (1968) Methicillinresistant Staphylococcus aureus at Boston City Hospital. Bacteriologic and epidemiologic observations. N Engl J Med 279:441-448

3. Bartels MD, Boye K, Rhod LA, Skov R, Westh H (2007) Rapid increase of genetically diverse methicillin-resistant Staphylococcus aureus, Copenhagen, Denmark. Emerg Infect Dis 13:1533-1540

4. Blanc DS, Pittet D, Ruef C, Widmer AF, Muhlemann K, Petignat C, Harbarth S, Auckenthaler R, Bille J, Frei R, Zbinden R, Peduzzi R, Gaia V, Khamis H, Bernasconi E, Francioli P (2002) Epidemiology of methicillin-resistant Staphylococcus aureus: results of a nation-wide survey in Switzerland. Swiss Med Wkly 132:223-229

5. Bootsma MC, Diekmann O, Bonten MJ (2006) Controlling methicillin-resistant Staphylococcus aureus: quantifying the effects of interventions and rapid diagnostic testing. Proc Natl Acad Sci 103:5620-5625

6. Boyce JM (1998) Are the epidemiology and microbiology of methicillin-resistant Staphylococcus aureus changing? JAMA 279:623-624

7. British Dental Association (BDA) (2003) Advice sheet: infection control in dentistry. www.bda.org/advice/goodpractice.cfm

8. Buehlmann M, Frei R, Fenner L, Dangel M, Fluckiger U, Widmer AF (2008) Highly effective regimen for decolonization of methicillin-resistant Staphylococcus aureus carriers. Infect Control Hosp Epidemiol 29:510-516

9. Centers for Disease Control and Prevention (CDC) (2008) Overview of community-associated MRSA. www.cdc.gov/ncidod/ dhqp/ar_mrsa_ca.html

10. Cook HA, Furuya EY, Larson E, Vasquez G, Lowy FD (2007) Heterosexual transmission of community-associated methicillinresistant Staphylococcus aureus. Clin Infect Dis 44:410-413

11. Cooper BS, Stone SP, Kibbler CC, Cookson BD, Roberts JA, Medley GF, Duckworth G, Lai R, Ebrahim S (2004) Isolation measures in the hospital management of methicillin resistant Staphylococcus aureus (MRSA): systematic review of the literature. BMJ 329:533-538

12. David MZ, Glikman D, Crawford SE, Peng J, King KJ, Hostetler MA, Boyle-Vavra S, Daum RS (2008) What is community- associated methicillin-resistant Staphylococcus aureus? J Infect Dis 197:1235-1243

13. Didilescu AC, Skaug N, Marica C, Didilescu C (2005) Respiratory pathogens in dental plaque of hospitalized patients with chronic lung diseases. Clin Oral Investig 9:141-147

14. Diekema DJ, Pfaller MA, Schmitz FJ, Smayevsky J, Bell J, Jones RN, Beach M (2001) Survey of infections due to Staphylococcus species: frequency of occurrence and antimicrobial susceptibility of isolates collected in the United States, Canada, Latin America, Europe, and the Western Pacific region for the SENTRY Antimicrobial Surveillance Program, 1997-1999. Clin Infect Dis 32(Suppl 2):S114-S132

15. Fenner L, Widmer AF, Dangel M, Frei R (2008) Distribution of spa types among methicillin-resistant Staphylococcus aureus isolates during a 6 year period at a low-prevalence university hospital. J Med Microbiol 57:612-616

16. Fluit AC, Wielders CL, Verhoef J, Schmitz FJ (2001) Epidemiology and susceptibility of 3,051 Staphylococcus aureus isolates from 25 university hospitals participating in the European SENTRY study. J Clin Microbiol 39:3727-3732

17. Gordon RJ, Lowy FD (2008) Pathogenesis of methicillin-resistant Staphylococcus aureus infection. Clin Infect Dis 46(Suppl 5): $\mathrm{S} 350-\mathrm{S} 359$

18. Gorwitz RJ, Kruszon-Moran D, McAllister SK, McQuillan G, McDougal LK, Fosheim GE, Jensen BJ, Killgore G, Tenover FC, Kuehnert MJ (2008) Changes in the prevalence of nasal colonization with Staphylococcus aureus in the United States, 2001-2004. J Infect Dis 197:1226-1234

19. Humphreys H (2007) National guidelines for the control and prevention of methicillin-resistant Staphylococcus aureus-what do they tell us? Clin Microbiol Infect 13:846-853

20. Johnson AP, Aucken HM, Cavendish S, Ganner M, Wale MC, Warner M, Livermore DM, Cookson BD (2001) Dominance of EMRSA-15 and -16 among MRSA causing nosocomial bacteraemia in the UK: analysis of isolates from the European Antimicrobial Resistance Surveillance System (EARSS). J Antimicrob Chemother 48:143-144

21. Jorgensen JH (1991) Mechanisms of methicillin resistance in Staphylococcus aureus and methods for laboratory detection. Infect Control Hosp Epidemiol 12:14-19

22. Kazakova SV, Hageman JC, Matava M, Srinivasan A, Phelan L, Garfinkel B, Boo T, McAllister S, Anderson J, Jensen B, Dodson D, Lonsway D, McDougal LK, Arduino M, Fraser VJ, Killgore G, Tenover FC, Cody S, Jernigan DB (2005) A clone of methicillinresistant Staphylococcus aureus among professional football players. N Engl J Med 352:468-475

23. Kohn WG, Collins AS, Cleveland JL, Harte JA, Eklund KJ, Malvitz DM (2003) Guidelines for infection control in dental health-care settings-2003. MMWR 52(No.RR-17):1-61

24. Kollef MH, Micek ST (2006) Methicillin-resistant Staphylococcus aureus: a new community-acquired pathogen? Curr Opin Infect Dis 19:161-168

25. Kommission für Krankenhaushygiene und Infektionsprävention beim Robert Koch-Institut (2006) Infektionsprävention in der Zahnheilkunde- Anforderungen an die Hygiene. BundesgesundheitsblGesundheitsforsch- Gesundheitsschutz 49:375-394

26. Kurita H, Kurashina K, Honda T (2006) Nosocomial transmission of methicillin-resistant Staphylococcus aureus via the surfaces of the dental operatory. Br Dent J 201:297-300

27. Larsen A, Stegger M, Goering R, Sorum M, Skov R (2007) Emergence and dissemination of the methicillin resistant Staphylococcus aureus USA300 clone in Denmark (2000-2005). Euro Surveill 12(2): pii=682. Available online: www.eurosurveillance. org/ViewArticle.aspx?Articleid $=682$

28. Liassine N, Auckenthaler R, Descombes MC, Bes M, Vandenesch F, Etienne J (2004) Community-acquired methicillin-resistant 
Staphylococcus aureus isolated in Switzerland contains the Panton-Valentine leukocidin or exfoliative toxin genes. J Clin Microbiol 42:825-828

29. Lowy FD (1998) Staphylococcus aureus infections. N Engl J Med 339:520-532

30. Martin MV, Hardy P (1991) Two cases of oral infection by methicillin-resistant Staphylococcus aureus. Br Dent J 170:6364

31. Mertz D, Frei R, Jaussi B, Tietz A, Stebler C, Fluckiger U, Widmer AF (2007) Throat swabs are necessary to reliably detect carriers of Staphylococcus aureus. Clin Infect Dis 45:475-477

32. Naimi TS, LeDell KH, Como-Sabetti K, Borchardt SM, Boxrud DJ, Etienne J, Johnson SK, Vandenesch F, Fridkin S, O'Boyle C, Danila RN, Lynfield R (2003) Comparison of community- and health care-associated methicillin-resistant Staphylococcus aureus infection. JAMA 290:2976-2984

33. National Nosocomial Infections Surveillance (NNIS) System (2004) Data summary from January 1992 through June 2004, issued October 2004. Am J Infect Control 32:470-485

34. Renvert S, Lindahl C, Renvert H, Persson GR (2008) Clinical and microbiological analysis of subjects treated with Branemark or AstraTech implants: a 7-year follow-up study. Clin Oral Implants Res 19:342-347

35. Salgado CD, Farr BM, Calfee DP (2003) Community-acquired methicillin-resistant Staphylococcus aureus: a meta-analysis of prevalence and risk factors. Clin Infect Dis 36:131-139

36. Sherertz RJ, Bassetti S, Bassetti-Wyss B (2001) "Cloud" healthcare workers. Emerg Infect Dis 7:241-244
37. Shurland S, Zhan M, Bradham DD, Roghmann MC (2007) Comparison of mortality risk associated with bacteremia due to methicillin-resistant and methicillin-susceptible Staphylococcus aureus. Infect Control Hosp Epidemiol 28:273-279

38. Siegel JD, Rhinehart E, Jackson M, Chiarello L, the Healthcare Infection Control Practices Advisory Committee (2006) Management of multidrug-resistant organisms in healthcare settings. www.cdc.gov/ncidod/dhqp/pdf/ar/mdroGuideline2006.pdf

39. Smith AJ, Jackson MS, Bagg J (2001) The ecology of Staphylococcus species in the oral cavity. J Med Microbiol 50:940-946

40. Smith AJ, Robertson D, Tang MK, Jackson MS, MacKenzie D, Bagg J (2003) Staphylococcus aureus in the oral cavity: a threeyear retrospective analysis of clinical laboratory data. Br Dent $\mathrm{J}$ 195:701-703

41. Strausbaugh LJ, Siegel JD, Weinstein RA (2006) Preventing transmission of multidrug-resistant bacteria in health care settings: a tale of 2 guidelines. Clin Infect Dis 42:828-835

42. Tiemersma EW, Bronzwaer SL, Lyytikainen O, Degener JE, Schrijnemakers P, Bruinsma N, Monen J, Witte W, Grundman H (2004) Methicillin-resistant Staphylococcus aureus in Europe, 1999-2002. Emerg Infect Dis 10:1627-1634

43. van Trijp MJ, Melles DC, Hendriks WD, Parlevliet GA, Gommans M, Ott A (2007) Successful control of widespread methicillinresistant Staphylococcus aureus colonization and infection in a large teaching hospital in The Netherlands. Infect Control Hosp Epidemiol 28:970-975

44. Weber JT (2005) Community-associated methicillin-resistant Staphylococcus aureus. Clin Infect Dis 41(Suppl 4):S269-S272 